\title{
CONCENTRAÇÃO DE BIXINA E LIPÍDIOS EM SEMENTES DE URUCUM DA COLEÇÃO DO INSTITUTO AGRONÔMICO (IAC) (1)
}

\author{
PAULO ROBERTO NOGUEIRA CARVALHO $\left(2^{*}\right)$; MARTA GOMES DA SILVA $\left({ }^{2}\right)$; \\ ELIANE GOMES FABRI ( $\left.{ }^{3}\right)$; PAULO EDUARDO DA ROCHA TAVARES $\left({ }^{2}\right)$; \\ ANTÔNIO LÚCIO MELLO MARTINS $\left({ }^{4}\right)$; LEANDRO RODRIGO SPATTI $\left({ }^{4}\right)$
}

\begin{abstract}
RESUMO
Existe uma extensa variedade genética no urucum que são conhecidas por denominações como "focinho de rato", "cabeça de moleque", "peruana", "bico de pato", "amarela", "piave” entre outras. Todas são caracterizadas por propriedades como porte da planta, período de produção, forma e cores das cachopas, deiscência, etc. A última década tem sido marcada por um melhoramento genético, buscando maior produtividade e, principalmente, maior teor de pigmentos. O Instituto Agronômico (IAC) comporta no Polo Regional Centro Norte, (Pindorama, SP), uma coleção de plantas de urucum, com mais de 25 acessos, que em trabalhos anteriores foram avaliadas quanto às suas características agronômicas. Contudo, os teores de bixina e lipídeos, que também são características importantes das sementes de urucum, pois afetam diretamente o rendimento industrial, não foram estudados. Este estudo teve como objetivo a validação de uma metodologia analítica de determinação de bixina e a caracterização da coleção do IAC quanto aos teores de bixina e lipídios. Os resultados indicaram que no método proposto houve linearidade na faixa de trabalho utilizada para a análise de sementes de urucum. Os limites de detecção e quantificação foram de 0,31 e 0,63 g de carotenóides totais expressos como bixina por $100 \mathrm{~g}$ de semente, respectivamente. O método foi preciso mas sensível a alterações na massa de amostra, tempo de extração e no coeficiente de absorção $\left(\mathrm{E}_{1 \mathrm{~cm}}^{1 \%}\right)$. As árvores existentes na coleção possuem altos teores de bixina na semente, variando (em base seca) de 3,12 $\pm 0,06 \%$ a $6,26 \pm 0,06 \%$. Os teores de lipídios variaram de 1,97 $\pm 0 \%$ a 3,98 $\pm 0,09 \%$. Não foram observadas correlações entre as concentrações de bixina e de lipídios.
\end{abstract}

Palavras chave: urucum, bixina, lipídeos.

\section{ABSTRACT \\ BIXIN AND LIPIDS CONTENT IN ANNATO SEEDS FROM COLLECTION OF THE INSTITUTO AGRONÔMICO (IAC)}

Annato has wide genetic varieties that are known by different names including "focinho de rato", "cabeça de moleque", peruana, "bico de pato", yellow and piave. Different properties are used to characterize them, namely, size of plant, production period, shape and fruit color, dehiscence, etc. The last decade have been marked by genetic improvement researches, in order to reach greater productivity and, especially, higher pigment content. The IAC has at the Polo Regional Centro Norte, in Campinas-SP, a collection of annatto plants with more than 25 varieties, which in previous studies were evaluated regarding their agronomic characteristics. However, bixin and lipids levels were not studied and both characteristics of the annatto seeds are very important, once the industrial performance during dyes production may be directly affected. The aim of the present study was to validate a methodology for bixin determination in annatto seeds and to evaluate the IAC collection for bixin and lipids content. The proposed method showed a linear response for each compound analyzed in the work concentration range. The detection and quantification limits for the bixin, were, respectively 0.31 and $0.63 \mathrm{~g}$ per $100 \mathrm{~g}$ seeds. The repeatability study

(1) Recebido para publicação em 24 de junho de 2009 e aceito em 11 de fevereiro de 2010.

(2) Instituto de Tecnologia de Alimentos, Av. Brasil, 2880, 13070-178 Campinas (SP).E-mail: carvalho@ital.sp.gov.br $\left(^{*}\right)$ Autor correspondente; martags@ital.sp.gov.br

(3) Instituto Agronômico, Centro de Horticultura, Campinas (SP). E-mail: efabri@iac.sp.gov.br

(4) Polo Regional Centro-Norte, Pindorama (SP). 
revealed a precise method; thus, significant variations have occurred when some parameters are changed, such as sample amount, time of extraction and absorption coefficient $\left(\mathrm{E}_{1 \mathrm{~cm}}^{1 \%}\right)$. The trees in the collection exhibited relatively high levels of bixin in the seeds, ranging (on dried basis) from 3,12 $\pm 0,06 \%$ to $6,26 \pm 0,06 \%$. The levels of lipid varied from $1,97 \pm 0 \%$ to $3,98 \pm 0,09 \%$. No correlation was observed between bixin and lipids concentrations.

Key words: Annato, bixin, lipids.

A planta do urucum é um arbusto de rápido crescimento, que alcança de 4 a 6 metros de altura, de aspecto robusto com flores vistosas cuja cor depende da variedade (PINEDA, 2003). Os frutos ou cachopas são cápsulas de duas partes, que contém de 30 a 45 sementes, podendo ser ovóides, cordiformes, esféricos e, em alguns casos, achatados. As cachopas podem chegar a até $5 \mathrm{~cm}$ de largura, glabas ou pilosas e sua coloração varia do verde ao vermelho intenso. O cultivo perene tem boas perspectivas em programas agrícolas, principalmente destinados a pequenos e médios produtores (MAZZANI, 2000). MAZZANI analisou 10 variedades diferentes de urucum em uma coleção instalada na Venezuela e concluiu que o grau de diferença existente entre as plantas é dependente de sua procedência. Estas análises revelam que estes recursos podem ser utilizados como fonte de germoplasma para trabalhos de melhoramento.

Existe uma extensa variedade genética no urucum que são conhecidas com denominações como "focinho de rato", "cabeça de moleque", "peruana", "bico de pato", "amarela", "Wagner" e "Piave", entre outras. Todas são caracterizadas por propriedades como porte da planta, período de produção, forma e cores das cachopas, deiscência, etc. (BALIANE, 1982).

A última década tem sido marcada por um melhoramento genético da espécie, buscando maior produtividade e, principalmente, maior teor de pigmentos. A concentração desses pigmentos, expressos geralmente como bixina, superou a média de $2,5 \%$ e a produtividade já atinge valores superiores a $1500 \mathrm{~kg} \mathrm{ha}^{-1}$ (CARvalho et al., 2007).

A inflorescência do urucum geralmente tem início dos 18 aos 21 meses após o plantio. A colheita das cachopas ocorre em dois períodos, a primeira de fevereiro e março a segunda em agosto e setembro. Após a colheita, geralmente realizada manualmente, as cachopas são secas e, em seguida, beneficiadas. $\mathrm{O}$ beneficiamento é realizado manualmente ou por meio de máquinas apropriadas para esta finalidade (CARVALHO e HeIn, 1989).

Segundo KaTo et al. (1992) o ponto máximo de qualidade fisiológica com base na porcentagem e no índice de velocidade de emergência, situou-se entre 72 e
79 dias após a antese da primeira flor da inflorescência. As porcentagens de bixina acima de 2,5\% só foram observadas nas sementes colhidas entre 30 e 51 dias. As épocas correspondentes aos estádios de melhor qualidade fisiológica não coincidiram com as de maiores porcentagens de corantes.

$\mathrm{Na}$ semente de urucum, observa-se em sua superfície um arilo que possui várias substâncias além do pigmento vermelho característico. Este arilo representa cerca de $5 \%$ a $10 \%$ do peso da semente, dos quais apenas $30 \%$ são representados pelo carotenóide bixina. Os 70\% restantes estão divididos em cinzas $(2,0 \%)$, proteínas $(2,5 \%)$, lipídeos $(30 \%)$, carboidratos $(32 \%)$ e umidade $(3,5 \%)$. A semente sem o arilo possui 9,8\% de umidade, 4,6\% de cinzas, $10,8 \%$ de proteína, 4,8\% de lipídeos e 70\% de carboidratos (CARVALHO et al., 1991).

Atualmente, uma das maiores dificuldades na comparação entre os resultados do teor de bixina de diferentes procedências está na falta de uniformidade dos métodos de análise e da ausência de demonstração da adequação desses mesmos métodos aos seus propósitos. As metodologias usadas variam desde a utilização de diferentes soluções para a extração do pigmento até o uso inadequado dos coeficientes de absorção $\left(E_{1 \mathrm{~cm}}^{1 \%}\right)$ para a quantificação dos carotenóides. Carvalho et al. (1993), publicaram um estudo indicando os coeficientes de absorção adequados para cada tipo de extrato (bixina ou sal de norbixina) e a possibilidade de degradação do pigmento durante o processo analítico.

Diante do exposto, o objetivo deste trabalho foi a validação de uma metodologia analítica de determinação de bixina e a caracterização da coleção do IAC quanto aos teores de bixina e lipídios.

No período de 28 a 30 de maio de 2007, logo após uma capina, foi realizada uma amostragem das cachopas das plantas de cada acesso de urucum do banco de germoplasma do IAC, localizado no Polo Regional Centro-Norte, no município de Pindorama, SP. Cada acesso esteve representado por uma amostra (cachopas) de, no mínimo, cinco plantas distribuídas aleatoriamente na coleção (Figura 1). As cachopas foram colhidas verdes, envasadas em sacos de polietileno (raschel), identificadas e encaminhadas para o Instituto 
Tabela 1. Resultados das análises de bixina, lipídeos e umidade das sementes da coleção da APTA Regional Centro-Norte. $\bar{X}=$ Média de três repetições analíticas, simultâneas e independentes; s = Estimativa de desvio-padrão; na = não analisada

\begin{tabular}{|c|c|c|c|c|c|c|c|c|}
\hline \multirow[t]{2}{*}{ Amostras } & \multicolumn{3}{|c|}{$\begin{array}{l}\text { Bixina (\%) } \\
\text { Base seca }\end{array}$} & \multicolumn{3}{|c|}{$\begin{array}{l}\text { Lipídeos (\%) } \\
\text { Base seca }\end{array}$} & \multicolumn{2}{|c|}{ Umidade (\%) } \\
\hline & $\bar{X}$ & $\mathrm{~s}$ & $*$ & $\bar{X}$ & $\mathrm{~s}$ & $*$ & $\bar{X}$ & $\mathrm{~s}$ \\
\hline 1 & 4,23 & 0,08 & abcd & 2,34 & 0,06 & $a b$ & 6,85 & 0,01 \\
\hline 2 & 4,18 & 0,07 & abcd & 2,90 & 0,04 & $\mathrm{ec}$ & 5,96 & 0,42 \\
\hline 3 & 3,82 & 0,24 & aef & 2,36 & 0,04 & $a b$ & 5,31 & 0,17 \\
\hline 4 & 3,80 & 0,06 & aefg & 2,84 & 0,04 & de & 5,76 & 0,21 \\
\hline 6 & 3,12 & 0,06 & $\mathrm{~h}$ & 2,82 & 0,07 & de & 4,27 & 1,57 \\
\hline 9 & 3,94 & 0,09 & $a b f$ & 2,70 & 0,07 & de & 4,11 & 0,14 \\
\hline 11 & 3,75 & 0,07 & aefg & 2,30 & 0,01 & $\mathrm{ab}$ & 4,69 & 0,84 \\
\hline 12 & 3,28 & 0,04 & egh & 2,28 & 0,04 & $\mathrm{ab}$ & 5,34 & 0,26 \\
\hline 13 & 3,30 & 0,04 & egh & 3,39 & 0,02 & $\mathrm{f}$ & 6,05 & 0,04 \\
\hline 14 & 4,46 & 0,11 & bcd & 2,80 & 0,06 & de & 4,73 & 0,09 \\
\hline 15 & 3,25 & 0,10 & gh & 3,40 & 0,03 & $f$ & 4,60 & 0,03 \\
\hline 16 & 3,38 & 0,09 & egh & na & na & na & 4,81 & 1,41 \\
\hline 17 & 4,64 & 0,11 & di & 2,69 & 0,06 & de & 3,55 & 0,40 \\
\hline 18 & 5,38 & 0,02 & $\mathrm{jk}$ & 3,33 & 0,11 & $\mathrm{f}$ & 3,79 & 0,39 \\
\hline 19 & 3,63 & 0,08 & efgh & 3,15 & 0,04 & $\mathrm{cf}$ & 6,83 & 0,60 \\
\hline 21 & 3,79 & 0,12 & aefg & 2,82 & 0,02 & de & 2,86 & 0,18 \\
\hline 22 & 5,49 & 0,09 & $\mathrm{jk}$ & 3,27 & 0,04 & $\mathrm{f}$ & 8,56 & 0,88 \\
\hline 23 & 3,95 & 0,22 & $\mathrm{abf}$ & 2,77 & 0 & de & 5,01 & 1,39 \\
\hline 24 & 3,98 & 0,13 & $a b c f$ & 2,72 & 0,01 & de & 3,50 & 0,35 \\
\hline 26 & 3,60 & 0,04 & efgh & 2,71 & 0,03 & de & 3,35 & 0,35 \\
\hline 27 & 5,67 & 0,03 & $\mathrm{k}$ & 3,98 & 0,09 & $\mathrm{~g}$ & 6,68 & 0,51 \\
\hline 28 & 3,71 & 0,02 & aefg & 2,63 & 0,02 & $\mathrm{~d}$ & 6,30 & 0,78 \\
\hline 29 & 4,52 & 0,10 & cdi & 2,87 & 0,09 & de & 5,87 & 1,24 \\
\hline 31 & 5,07 & 0,09 & $\mathrm{ij}$ & 2,89 & 0,10 & $\mathrm{e}$ & 7,47 & 0,28 \\
\hline 32 & 4,39 & 0,13 & bcd & 1,97 & 0 & $\mathrm{~h}$ & 7,04 & 0,57 \\
\hline 34 & 6,26 & 0,06 & 1 & 2,27 & 0,11 & $\mathrm{a}$ & 5,53 & 0,19 \\
\hline
\end{tabular}

* As amostras com a mesma letra, na mesma coluna, não apresentam diferenças significativas (as médias foram comparadas entre si pelo teste de Tukey com $5 \%$ de probabilidade de erro).

de Tecnologia de Alimentos para a secagem (ao sol), retirada das sementes e análises.

O método utilizado tem como princípio a saponificação da bixina em sal de norbixina, que na forma hidrossolúvel foi quantificado por espectrofotometria em comprimento de onda na região do visível, conforme descrito a seguir: Para cada $10 \mathrm{~g} \pm 0,2 \mathrm{~g}$ de sementes de urucum foram adicionados $60 \mathrm{~mL}$ da solução de extração $\left({ }^{5}\right)$, a mistura foi aquecida até a ebulição por 2 minutos e a solução, esfriada à temperatura ambiente. $\mathrm{O}$ extrato (e as sementes) foram pesados e a massa corrigida para $250 \mathrm{~g}$ com água destilada. A mistura foi agitada por 10 minutos, uma alíquota de $1 \mathrm{~mL}$ foi transferida para um balão volumétrico de $100 \mathrm{~mL}$ e o volume completado com solução de KHO 0,5\% (m/v). A amostra foi agitada e nova alíquota foi diluída em $\mathrm{KOH}$ $0,5 \%(\mathrm{~m} / \mathrm{v})$ para obter leitura espectrofotométrica entre 0,3 e 0,7 unidades de absorbância. A leitura foi realizada em um comprimento de onda de $453 \mathrm{~nm}$. Foi utilizada, como referência, uma solução de $\mathrm{KOH} 0,5 \%$ (m/v). A conversão do sal de norbixina para bixina foi realizada multiplicando o resultado analítico por 1,16.

A metodologia foi validada quanto à linearidade, pela construção de uma curva de calibração de carotenóides totais expressos como "sal de norbixina" com seis pontos, considerando a origem; sensibilidade, pormeioda construçãodeuma curva padrãosuplementar com três pontos, com concentrações inferiores ao último ponto da curva de linearidade, lidos em triplicatas (Quattrocchi et al., 1992). Foram utilizadas as seguintes

(5) Solução de extração: 290 mL de óleo de mamona em 100 mL de hidróxido de potássio 45\% (m/v) e 190 mL de água destilada. Aquecer em bico de Bunsen até a mistura tornar-se clara. Retirar uma alíquota de $50 \mathrm{~mL}$ de solução sabão e adicionar 130 mL de solução de hidróxido de potássio 45\% (m/v) e 1000 mL de água deionizada. 


\begin{tabular}{|c|c|c|c|c|c|c|c|c|c|c|c|c|c|c|c|c|c|c|c|c|c|c|c|c|c|c|c|}
\hline $\begin{array}{l}24 \\
F \\
22 \\
F \\
F \\
19\end{array}$ & $\stackrel{N}{+}$ & $\begin{array}{l}F \\
F \\
F \\
28 \\
F \\
F\end{array}$ & $\underset{\infty}{N}$ & $\begin{array}{c}126 \\
F \\
F \\
F \\
F \\
121\end{array}$ & $\stackrel{N}{\omega}$ & $\begin{array}{l}127 \\
128 \\
129 \\
130 \\
131 \\
132\end{array}$ & 10. & $\begin{array}{l}F \\
F \\
F \\
F \\
F \\
F\end{array}$ & $\approx$ & $\begin{array}{l}F \\
F \\
F \\
F \\
F \\
F\end{array}$ & $\grave{N}$ & $\begin{array}{l}F \\
F \\
F \\
F \\
F \\
F\end{array}$ & $\stackrel{\omega}{N}$ & $\begin{array}{c}F \\
F \\
F \\
346 \\
347 \\
348\end{array}$ & $\infty$ & $\begin{array}{c}450 \\
F \\
F \\
447 \\
446 \\
445\end{array}$ & $\stackrel{N}{\circ}$ & $\begin{array}{c}F \\
452 \\
453 \\
454 \\
455 \\
456\end{array}$ & $\stackrel{N}{N}$ & $\begin{array}{c}558 \\
F \\
556 \\
F \\
F \\
553\end{array}$ & $\stackrel{\omega}{\omega}$ & $\begin{array}{c}559 \\
F \\
561 \\
F \\
F \\
564\end{array}$ & जั & $\begin{array}{l}666 \\
665 \\
664 \\
663 \\
662 \\
661\end{array}$ & $\stackrel{N}{\alpha}$ & $\begin{array}{c}667 \\
668 \\
669 \\
670 \\
671 \\
F\end{array}$ & 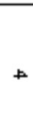 \\
\hline $\begin{array}{l}F \\
17 \\
16 \\
F \\
14 \\
13\end{array}$ & $\stackrel{\omega}{\sim}$ & $\begin{array}{l}31 \\
32 \\
F \\
F \\
35 \\
36\end{array}$ & $\stackrel{N}{\sim}$ & $\begin{array}{l}F \\
F \\
F \\
F \\
F \\
F\end{array}$ & $r$ & $\begin{array}{l}133 \\
134 \\
135 \\
136 \\
137 \\
138\end{array}$ & 10 & $\begin{array}{c}F \\
F \\
F \\
225 \\
F \\
223\end{array}$ & $\omega$ & $\begin{array}{l}241 \\
242 \\
243 \\
244 \\
245 \\
246\end{array}$ & 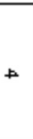 & $\begin{array}{c}336 \\
F \\
F \\
F \\
F \\
F\end{array}$ & 10. & $\begin{array}{l}F \\
F \\
F \\
F \\
F \\
F\end{array}$ & 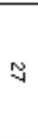 & $\begin{array}{l}444 \\
443 \\
442 \\
441 \\
440 \\
439\end{array}$ & $\stackrel{\omega}{\sim}$ & $\begin{array}{c}457 \\
F \\
459 \\
F \\
F \\
F\end{array}$ & + & $\begin{array}{c}F \\
F \\
550 \\
F \\
F \\
F\end{array}$ & $\stackrel{N}{\sim}$ & $\begin{array}{l}F \\
F \\
F \\
F \\
F \\
F\end{array}$ & $\mathbb{y}$ & $\begin{array}{c}660 \\
659 \\
658 \\
657 \\
F \\
F\end{array}$ & 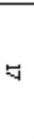 & $\begin{array}{l}673 \\
674 \\
675 \\
676 \\
677 \\
678\end{array}$ & $\vec{N}$ \\
\hline $\begin{array}{l}12 \\
F \\
F \\
9 \\
8 \\
7\end{array}$ & 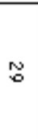 & $\begin{array}{l}37 \\
F \\
F \\
40 \\
F \\
42\end{array}$ & $\begin{array}{l}\omega \\
+\end{array}$ & $\begin{array}{c}\mathrm{F} \\
\mathrm{F} \\
\mathrm{F} \\
\mathrm{F} \\
\mathrm{F} \\
109\end{array}$ & ち & $\begin{array}{c}139 \\
F \\
141 \\
F \\
143 \\
F\end{array}$ & $\stackrel{\sim}{\sim}$ & $\begin{array}{l}F \\
F \\
F \\
F \\
F \\
F\end{array}$ & $\vec{\omega}$ & $\begin{array}{c}247 \\
248 \\
248 \\
250 \\
F \\
252\end{array}$ & $\stackrel{N}{\leftarrow}$ & $\begin{array}{c}\mathrm{F} \\
\mathrm{F} \\
\mathrm{F} \\
\mathrm{F} \\
\mathrm{F} \\
325\end{array}$ & $\Xi$ & $\begin{array}{c}F \\
F \\
F \\
F \\
F \\
360\end{array}$ & $E$ & $\begin{array}{c}438 \\
437 \\
F \\
F \\
F \\
433\end{array}$ & $\omega$ & $\begin{array}{c}F \\
F \\
F \\
F \\
F \\
468\end{array}$ & $\leftleftarrows$ & $\begin{array}{c}546 \\
F \\
544 \\
543 \\
542 \\
F\end{array}$ & $\begin{array}{l}N \\
+\end{array}$ & $\begin{array}{c}\mathrm{F} \\
\mathrm{F} \\
\mathrm{F} \\
\mathrm{F} \\
\mathrm{F} \\
576\end{array}$ & $\stackrel{N}{\infty}$ & $\begin{array}{c}F \\
653 \\
F \\
651 \\
650 \\
649\end{array}$ & $\stackrel{N}{\stackrel{N}{*}}$ & $\begin{array}{c}\mathrm{F} \\
\mathrm{F} \\
681 \\
\mathrm{~F} \\
\mathrm{~F} \\
684\end{array}$ & 10 \\
\hline $\begin{array}{l}6 \\
5 \\
4 \\
3 \\
2 \\
1\end{array}$ & \multirow[t]{6}{*}{$\omega$} & $\begin{array}{l}43 \\
44 \\
45 \\
46 \\
47 \\
48\end{array}$ & $\stackrel{\infty}{\infty}$ & $\begin{array}{c}F \\
107 \\
F \\
F \\
104 \\
103\end{array}$ & 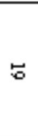 & $\begin{array}{c}145 \\
F \\
147 \\
148 \\
F \\
F\end{array}$ & $\stackrel{N}{\alpha}$ & $\begin{array}{c}216 \\
215 \\
214 \\
F \\
212 \\
211\end{array}$ & ${ }_{\infty}^{N}$ & $\begin{array}{c}F \\
F \\
F \\
F \\
257 \\
258\end{array}$ & $\stackrel{N}{\underset{\sim}{*}}$ & $\begin{array}{l}324 \\
323 \\
322 \\
321 \\
320 \\
319\end{array}$ & $N$ & $\begin{array}{c}361 \\
F \\
F \\
F \\
365 \\
366\end{array}$ & 5 & $\begin{array}{c}F \\
F \\
430 \\
429 \\
428 \\
427\end{array}$ & $\stackrel{N}{\sim}$ & $\begin{array}{c}469 \\
F \\
F \\
F \\
F \\
F\end{array}$ & $\stackrel{\omega}{N}$ & $\begin{array}{c}F \\
F \\
538 \\
F \\
F \\
F\end{array}$ & $\stackrel{N}{\sim}$ & $\begin{array}{c}577 \\
578 \\
579 \\
F \\
581 \\
F\end{array}$ & $\omega$ & $\begin{array}{c}648 \\
647 \\
F \\
645 \\
644 \\
F\end{array}$ & ち & $\begin{array}{l}F \\
F \\
F \\
F \\
F \\
F\end{array}$ & 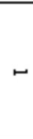 \\
\hline & & $\begin{array}{l}49 \\
F \\
51 \\
F \\
53 \\
54\end{array}$ & b & $\begin{array}{c}102 \\
F \\
100 \\
F \\
F \\
F\end{array}$ & $N$ & $\begin{array}{c}151 \\
152 \\
F \\
154 \\
F \\
156\end{array}$ & $\stackrel{N}{N}$ & $\begin{array}{c}210 \\
209 \\
F \\
F \\
F \\
205\end{array}$ & 5 & $\begin{array}{l}259 \\
260 \\
261 \\
262 \\
263 \\
264\end{array}$ & $N$ & $\begin{array}{c}F \\
F \\
316 \\
315 \\
314 \\
313\end{array}$ & $\underset{N}{N}$ & $\begin{array}{c}F \\
F \\
369 \\
F \\
F \\
F\end{array}$ & 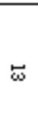 & $\begin{array}{c}F \\
F \\
424 \\
423 \\
422 \\
F\end{array}$ & $N$ & $\begin{array}{c}F \\
F \\
F \\
478 \\
479 \\
F\end{array}$ & 文 & $\begin{array}{c}534 \\
F \\
F \\
F \\
F \\
F\end{array}$ & b & $\begin{array}{c}F \\
584 \\
F \\
F \\
F \\
F\end{array}$ & 5 & $\begin{array}{c}F \\
F \\
640 \\
F \\
638 \\
637\end{array}$ & $a$ & $\begin{array}{c}F \\
692 \\
693 \\
694 \\
695 \\
F\end{array}$ & $\stackrel{N}{\sim}$ \\
\hline & & $\begin{array}{l}55 \\
56 \\
57 \\
58 \\
59 \\
60\end{array}$ & 它 & $\begin{array}{l}96 \\
95 \\
94 \\
93 \\
92 \\
91\end{array}$ & $\vec{N}$ & $\begin{array}{l}157 \\
158 \\
159 \\
160 \\
161 \\
162\end{array}$ & $\underset{\sim}{\omega}$ & $\begin{array}{l}204 \\
203 \\
202 \\
201 \\
200 \\
199\end{array}$ & $\stackrel{\infty}{\infty}$ & $\begin{array}{c}F \\
266 \\
267 \\
268 \\
269 \\
270\end{array}$ & $\stackrel{N}{\circ}$ & $\begin{array}{l}312 \\
311 \\
310 \\
309 \\
308 \\
307\end{array}$ & $b$ & $\begin{array}{c}F \\
F \\
375 \\
376 \\
377 \\
378\end{array}$ & $\cong$ & $\begin{array}{c}420 \\
419 \\
418 \\
F \\
F \\
415\end{array}$ & $\begin{array}{l}\omega \\
\leftarrow\end{array}$ & $\begin{array}{c}F \\
482 \\
483 \\
484 \\
F \\
F\end{array}$ & $\underset{\leftarrow}{N}$ & $\begin{array}{c}F \\
527 \\
526 \\
525 \\
F \\
523\end{array}$ & 10. & $\begin{array}{c}F \\
F \\
F \\
592 \\
593 \\
F\end{array}$ & $\stackrel{N}{\circ}$ & $\begin{array}{l}F \\
635 \\
634 \\
633 \\
632 \\
631\end{array}$ & 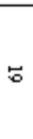 & $\begin{array}{c}697 \\
698 \\
699 \\
700 \\
F \\
702\end{array}$ & $\stackrel{N}{\omega}$ \\
\hline & & $\begin{array}{l}61 \\
62 \\
63 \\
64 \\
65 \\
66\end{array}$ & $\stackrel{5}{+}$ & $\begin{array}{l}90 \\
89 \\
88 \\
87 \\
86 \\
85\end{array}$ & $\vec{\omega}$ & $\begin{array}{c}63 \\
F \\
165 \\
166 \\
167 \\
168\end{array}$ & 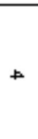 & $\begin{array}{l}198 \\
197 \\
196 \\
195 \\
194 \\
193\end{array}$ & $\stackrel{\infty}{\not}$ & $\begin{array}{c}271 \\
272 \\
273 \\
274 \\
275 \\
\mathrm{~F}\end{array}$ & $\stackrel{\sim}{\sim}$ & $\begin{array}{c}306 \\
F \\
304 \\
303 \\
302 \\
F\end{array}$ & $\stackrel{N}{\sim}$ & $\begin{array}{l}379 \\
380 \\
381 \\
382 \\
383 \\
384\end{array}$ & $\stackrel{N}{\alpha}$ & $\begin{array}{l}414 \\
413 \\
412 \\
411 \\
410 \\
409\end{array}$ & $\underset{+}{N}$ & $\begin{array}{l}487 \\
488 \\
489 \\
490 \\
491 \\
492\end{array}$ & $\mathbb{N}$ & $\begin{array}{l}522 \\
521 \\
520 \\
519 \\
518 \\
517\end{array}$ & 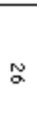 & $\begin{array}{l}595 \\
596 \\
597 \\
598 \\
599 \\
600\end{array}$ & $N$ & $\begin{array}{l}630 \\
629 \\
628 \\
627 \\
626 \\
625\end{array}$ & 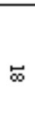 & $\begin{array}{l}703 \\
704 \\
705 \\
706 \\
707 \\
708\end{array}$ & $N$ \\
\hline & & $\begin{array}{l}67 \\
68 \\
69 \\
70 \\
71 \\
72\end{array}$ & $\mathscr{y}$ & $\begin{array}{l}84 \\
83 \\
82 \\
81 \\
80 \\
79\end{array}$ & $N$ & $\begin{array}{l}169 \\
170 \\
171 \\
172 \\
173 \\
174\end{array}$ & 学 & $\begin{array}{l}192 \\
191 \\
190 \\
189 \\
188 \\
187\end{array}$ & 点 & $\begin{array}{c}\mathrm{F} \\
\mathrm{F} \\
279 \\
\mathrm{~F} \\
\mathrm{~F} \\
\mathrm{~F}\end{array}$ & $y$ & $\begin{array}{c}\mathrm{F} \\
\mathrm{F} \\
\mathrm{F} \\
297 \\
\mathrm{~F} \\
\mathrm{~F}\end{array}$ & - & $\begin{array}{c}\mathrm{F} \\
\mathrm{F} \\
387 \\
388 \\
\mathrm{~F} \\
390\end{array}$ & 10 & $\begin{array}{c}408 \\
407 \\
F \\
405 \\
404 \\
F\end{array}$ & 它 & $\begin{array}{l}493 \\
494 \\
495 \\
496 \\
497 \\
498\end{array}$ & $\stackrel{N}{\omega}$ & $\begin{array}{l}516 \\
515 \\
514 \\
513 \\
512 \\
511\end{array}$ & $N$ & $\begin{array}{l}601 \\
602 \\
603 \\
604 \\
605 \\
606\end{array}$ & 0 & $\begin{array}{l}624 \\
623 \\
622 \\
621 \\
620 \\
619\end{array}$ & $\stackrel{\iota}{\omega}$ & $\begin{array}{c}709 \\
F \\
F \\
712 \\
713 \\
714\end{array}$ & $\underset{N}{\omega}$ \\
\hline & & & & $\begin{array}{l}78 \\
77 \\
76 \\
75 \\
74 \\
73\end{array}$ & $\stackrel{N}{\circ}$ & $\begin{array}{l}175 \\
176 \\
177 \\
178 \\
179 \\
180\end{array}$ & $\stackrel{\sim}{+}$ & $\begin{array}{c}186 \\
185 \\
184 \\
183 \\
182 \\
F\end{array}$ & $\stackrel{\omega}{\omega}$ & $\begin{array}{c}283 \\
284 \\
285 \\
286 \\
F \\
288\end{array}$ & ज酒 & $\begin{array}{c}F \\
293 \\
292 \\
291 \\
F \\
289\end{array}$ & 学 & $\begin{array}{c}391 \\
392 \\
393 \\
F \\
F \\
F\end{array}$ & $\widetilde{\omega}$ & $\begin{array}{l}402 \\
401 \\
400 \\
399 \\
398 \\
397\end{array}$ & $\stackrel{\infty}{\infty}$ & $\begin{array}{c}499 \\
500 \\
501 \\
502 \\
F \\
504\end{array}$ & 10 & $\begin{array}{c}510 \\
F \\
508 \\
507 \\
506 \\
505\end{array}$ & $r$ & $\begin{array}{l}607 \\
608 \\
609 \\
610 \\
611 \\
612\end{array}$ & $\vec{E}$ & $\begin{array}{c}618 \\
617 \\
616 \\
615 \\
614 \\
F\end{array}$ & $\stackrel{\infty}{+}$ & $\begin{array}{c}715 \\
716 \\
717 \\
718 \\
F \\
F\end{array}$ & $N$ \\
\hline
\end{tabular}

Figura 1. Mapa do banco de germoplasma de urucum do Instituto Agronômico, localizado no Polo Regional Centro-Norte, no município de Pindorama (SP). F = Falhas.

equações: Limite de deteç̧ão $=\left[\frac{Y_{b l}+{ }_{b l}^{3 S}}{b}\right]\left(\begin{array}{c}1 \\ \sqrt{n}\end{array}\right)$ e Limite de quantificação $=\left[\frac{Y_{b l}+{ }_{b l}^{10 S}}{b}\right]\left(\begin{array}{c}1 \\ \sqrt{n}\end{array}\right)$,

em que: $Y_{\mathrm{b} 1}$ é a estimativa da resposta do branco (coeficiente linear obtido pela equação da curva de calibração construída com a média das absorbância das amostras); $S_{\mathrm{b} 1}$ é a estimativa de desvio do padrão do branco (coeficiente linear obtido pela equação da curva de calibração construída com a estimativa de desviopadrão das médias das absorbância das amostras); $b$ é o coeficiente angular da curva analítica e n é o número de pontos da curva. A precisão foi avaliada pela realização de sete repetições do método analítico em uma mesma amostra, utilizando a equação de Horwitz (Horwitz, 1982) como referência. Para a avaliação da robustez do método, foi utilizado um planejamento fatorial saturado $\left(2^{7-4}\right)$ com a variação dos seguintes fatores: massa da amostra (2,5 g, $5 \mathrm{~g}, 10 \mathrm{~g}$ e $25 \mathrm{~g})$, tempo de agitação (5 e 10 minutos), tempo de ebulição (1 e 2 minutos) e $\mathrm{E}_{1 \mathrm{~cm}}^{1 \%}(2850$ a $453 \mathrm{~nm}$ e 2870 a $482 \mathrm{~nm})$. Os valores resultantes foram comparados com a estimativa de desvio-padrão observada no estudo da precisão do método (QuATtrocchi et al., 1992). Considerou-se que houve diferença significativa ao nível de $95 \%$ quando o valor absoluto do resultado foi maior que a estimativa de desvio-padrão constatada no estudo da precisão do método, multiplicado por raiz de 2 .

Os lipídeos foram analisados segundo StoldWeibull (DiEMAIR, 1963), cujo teor de substâncias solúveis em éter é determinado gravimetricamente, após destruição das proteínas, carboidratos e outros componentes, com ácido clorídrico.

Para o estudo da linearidade do método foi construída uma curva com concentrações crescentes de sal de norbixina (Figura 2). A análise estatística 
dos resultados indicou que o método representa uma correlação significativa, com uma probabilidade de $95 \%$. Portanto o método foi considerado linear para a faixa de trabalho. Os limites de detecção e de quantificação foram, respectivamente, 0,31 e 0,62 g de carotenóides totais expressos como "sal de norbixina" por $100 \mathrm{~g}$ de sementes de urucum. Esse resultado é muito inferior aos valores geralmente encontrados nas sementes de urucum, indicando que o método é sensível suficientemente para esse tipo de análise.

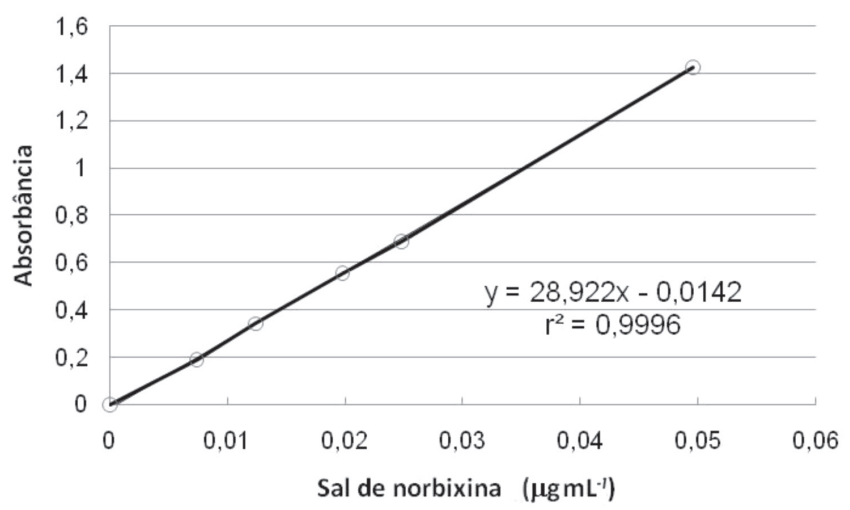

Figura 2. Absorbância da solução de sal de norbixina para diferentes concentrações.

Para o cálculo da precisão do método analítico, foram realizados sete repetições analíticas de uma mesma amostra, apresentando como resultado 4,71 $\pm 0,09$ g de carotenóides totais expressos como "sal de norbixina" por $100 \mathrm{~g}$ de sementes de urucum. O coeficiente de variação $(\mathrm{CV}=2 \%)$ foi inferior aos coeficientes calculados pela equação de Horwitz $(\mathrm{CV}=$ $4 \%$ ), demonstrando boa precisão do método analítico.

$\mathrm{Na}$ avaliação da robustez do método foi possível verificar que houve diferença significativa quando se variou os seguintes fatores: a massa da amostra de $10 \mathrm{~g}$ para 2,5 g, 5,0 g e 25 g; o tempo de agitação de 5 para 10 minutos e a substituição do $\mathrm{E}_{1 \mathrm{~cm}}^{1 \%} 2850$ a $453 \mathrm{~nm}$ por $\mathrm{E}_{1 \mathrm{~cm}}^{1 \%}$ 2870 a $482 \mathrm{~nm}$.

Com base nesses resultados, ficou estabelecida para o método analítico uma massa de amostra inicial de $10 \mathrm{~g} \pm 0,2 \mathrm{~g}$; um tempo de agitação de 10 minutos e o uso de comprimento de onda de $453 \mathrm{~nm}$ com o $E_{1 \mathrm{~cm}}^{1 \%}=2850 \pm 40$.

No método validado verificou-se uma incerteza expandida de aproximadamente $6 \%(4,71 \mathrm{~g} \pm 0,28 \mathrm{~g}$ de carotenóides totais expressos como "sal de norbixina" por $100 \mathrm{~g}$ de sementes de urucum $)(\mathrm{k}=2)$.

As plantas da coleção de urucum do IAC, no Polo Regional Centro-Norte, já foram avaliadas, em trabalhos anteriores, quanto à produtividade, resistência às pragas, características dos frutos e das sementes (Bovi et al., 1994). Segundo os autores, o nível de deiscência e a forma do fruto não tiveram coeficiente de correlação significativo com a produção (massa de semente). O tamanho, a massa e o número de sementes por fruto proporcionaram correlação positiva, mas baixa.

$\mathrm{Na}$ tabela 1 estão relacionados os resultados das análises de bixina e lipídios (base seca) e umidade (sementes inteiras) das amostras da coleção. Os resultados indicaram que houve diferença significativa no resultado de bixina das árvores da coleção, variando de um mínimo de 3,12 $\pm 0,06 \%$ (Amostra 6) a um máximo de 6,26 $\pm 0,06 \%$ (Amostra 34). A maior parte das amostras se situou na faixa entre 3 e $4 \%$ (Amostras $3,4,9,11,12,13,15,16,19,21,23,24,26$ e 28). Nas amostras 1, 2, 14, 17, 29 e 32 apresentaram teores de bixina na faixa entre $4 \%$ e $5 \%$ e as amostras $18,22,27$ e 31 apresentaram teores de bixina entre $5 \%$ e $6 \%$. Nas amostra 34 foi a única a ter concentração de bixina superior a $6 \%$.

Os resultados de umidade variaram muito entre as sementes com um mínimo de 2,86\% (amostra 21) a um máximo de $8,56 \%$ (amostra 22). Como as análises foram realizadas com amostras de sementes inteiras, observou-se uma variação muito grande entre alguns ensaios, sugerindo falta de homogeneidade na umidade entre as diferentes amostras de sementes.

Os resultados de lipídios estão expressos em base seca e variaram de um mínimo de 1,97 $\pm 0 \%$ (amostra 32) a um máximo de 3,98 $\pm 0,09 \%$ (amostra 27). A maior parte ficou entre $2 \%$ e $3 \%$ (Amostras 34, 12, 11, 1, 3, 28, $17,9,26,24,23,14,21,6,4,29,31$ e 2). As amostras 19, $22,18,13,15$ e 27 tiveram teores de lipídios superiores a $3 \%$.

Não foram observadas correlações entre as concentrações de bixina e de lipídios, de bixina e umidade ou ainda de lipídios e umidade das sementes estudadas.

A amostra 34 foi a que teve os melhores resultados para utilização na indústria de corantes (extração alcalina). $\mathrm{O}$ alto teor de carotenóides $(6,26 \%)$ e o baixo teor de lipídeos $(2,27 \%)$ facilitam o processo de extração mais utilizado pelas indústrias de corante (saponificação).

$\mathrm{Na}$ amostra 27, apesar da elevada concentração de carotenóides $(5,67 \%)$, há também alta concentração de lipídeos $(3,98 \%)$. Esse alto teor de lipídeos pode dificultar a etapa de saponificação utilizada pelas indústrias de corantes. Todavia esse mesmo acesso pode ser interessante para indústrias que utilizam a extração do corante por meio de óleo vegetal. 
As sementes de urucum com teores menores que 2,5 gramas de carotenóides totais expressos como bixina por $100 \mathrm{~g}$ de sementes de urucum (base úmida) encontram dificuldades de comercialização, por diminuírem muito o rendimento industrial e a qualidade do produto final (corante). Nesse trabalho, nenhuma amostra apresentou valores, em base úmida, inferior a esse limite.

Nos acessos de urucum do banco de germoplasma do Instituto Agronômico (IAC), mantido no Polo Regional Centro-Norte, em Pindorama, (SP). Os resultados são bastante variáveis tanto para os teores de bixina, como de lipídios.

Em alguns acessos os teores de bixina são bastante interessantes, superior as média observada no comércio dessas sementes.

$\mathrm{Na}$ amostra 34 observam-se melhores resultados quando suas sementes são destinadas à extração de corantes por saponificação.

Não há correlação entre os teores de bixina e de lipídios nas amostras estudadas.

\section{REFERÊNCIAS}

BALIANE, A. A cultura do urucueiro. Rio de Janeiro: Empresa de Assistência Técnica e Extensão Rural, 1982, 10p.

BOVI, O.A.; PERECIN-CALHEIROS, M.B.; MAIA, N.B.; DUARTE, F.R.; MARTINS, A.L. Correlações fenotípicas em populações de urucum. Rendimento nos três primeiros anos de produção. In. CONGRESSO BRASILEIRO DE CORANTES NATURAIS, 2.; SIMPÓSIO BRASILEIRO DE URUCUM, 2. 1994, Belém. Resumos... Belém:

CARVALHO, P.R.N.; CARVALHO, C.R.L.; MANTOVANI, D.M.B. Estudo da composição de sementes, cachopas, folhas e galhos do urucueiro. In. SEMINÁRIO INTERNACIONAL DE CORANTES NATURAIS PARA ALIMENTO, 2., 1991, Campinas. Resumos... Campinas: ITAL, 1991. p317.

CARVALHO, P.R.N.; HEIN, M. Urucum: Uma fonte de corante natural. Coletânea do ITAL, v.19, p.25-33, 1989.

CARVALHO, P.R.N.; DA SILVA M.G.; MOREIRA, C.G.C. Avaliação dos Métodos Espectrofotométricos de Análise de Sementes de Urucum (Bixa orellana, L.). Coletânea do ITAL, v.23, p.181-188, 1993

CARVALHO, P.R.N.; TAVARES, P.E.R.; FABRI, E.G. REUNIÃO NACIONAL DA CADEIA PRODUTIVA DO URUCUM. Campinas. Resumos... Campinas, ITAL, 2007. 107p.

DIEMAIR, W. Laboratoriumsbuch fur Lebensmittelchemiker. Verlag Von Theodor Steinkopff: Dresden. 8 Auflage, 1963.

HORWITZ, W. Evaluation of analytical methods used for regulation of foods and drugs. Analytical Chemists, v.54, p.67A-76A, 1982.

KATO, O.R.; FIGUEIRÊDO, F.J.C.; BELFORT, A.J.L.; NOGUEIRA, O.L.; BARBOSA, W.C. Época de colheita de sementes de urucum. Pesquisa Agropecuária Brasileira, v.27, p.1291-1302, 1992.

MAZZANI, E.; MARÍN C.R.; SEGOVIA, V. Estudio de la variabilidad existente en la colección de onoto(Bixa orellana $\mathrm{L}$.) del CENIAP; FONAIAP; Revista Faculdad de Agronômica (LUZ), v.17, p.492-504, 2000.

PINEDA, J.E.D.; CALDERÓN, L.S. Planta piloto para obtener colorante de la semilla del achiote (Bixa orellana). Revista Universidad EAFIT, v.39, p.8-22, 2003.

QUATTROCCHI, O. A.; ANDRIZZI, S.A.; LABA, R.F Introdutión a la HPLC Aplicación y Práctica. Buenos Aires: Artes Gráficas Farro, 1992. 407p. 